\title{
Phases of quantum states in completely positive non-unitary evolution *
}

\author{
J. G. Peixoto de Faria and A. F. R. de Toledo Piza \\ Departamento de Física-Matemática, Instituto de Física, \\ Universidade de São Paulo, C.P. 66318, 05315-970, \\ São Paulo, São Paulo, Brazil \\ M. C. Nemes \\ Departamento de Física, Instituto de Ciências Exatas, \\ Universidade Federal de Minas Gerais, C.P. 702, 30161-970, \\ Belo Horizonte, Minas Gerais, Brazil
}

(Dated: October 31, 2018)

\begin{abstract}
We define an operational notion of phases in interferometry for a quantum system undergoing a completely positive non-unitary evolution. This definition is based on the concepts of quantum measurement theory. The suitable generalization of the Pancharatnan connection allows us to determine the dynamical and geometrical parts of the total phase between two states linked by a completely positive map. These results reduce to the known expressions of the total, dynamical and geometrical phases for pure and mixed states evolving unitarily.
\end{abstract}

PACS: 03.65.Vf, 03.65.Ta, 03.65.Yz

Introduction: The interest in the holonomy concept has considerably grown in physics in the last years. Such activity began with the publication of Berry's work [1]. Berry showed that the energy eigenfunctions accumalated a phase factor of purely geometrical origin during an adiabatic cyclic evolution. Simon 2] gave a mathematical interpretation of Berry's phase as the holonomy of a complex line bundle. Later, Wilczek and Zee [3] generalized this concept to non-abelian phases associated with cyclic evolution of degenerate hamiltonians in the adiabatic limit; Aharonov and Anandan [4] showed that the adiabatic limit is unnecessary and Samuel and Bhandari [5], using the ideas developed by Pancharatnam [6], showed that the Berry's phase arises even in noncyclic and non-unitary evolutions (see also Ref. [7]). These treatments of the geometric phases are restricted only to pure states, like the majority of the works on this topic. However, in a large number of applicatons (in particular, the Quantum Theory of Measurement 8] and the Theory of Quantum Open Systems [9]), the concept of state must be generalized to include statistical mixtures. The question of the mixed state holonomy was already studied by Uhlmann [10] in a purely mathematical context. Sjöqvist and co-workers [1] recently developed an operational concept of phases for mixed states undergoing unitary evolution using an interferometric model. Despite these achievements, the study of geometric phases remains still incipient particularly for mixed states undergoing non-unitary evolution, characteristic of quantum open systems.

In the present work we construct an operational definition of phases between mixed states undergoing completely positive (CP) non-unitary time evolution. We employed a procedure similar to that used by Sjöqvist et al. [11] in the context of unitarily evolving mixed states in interferometry. Moreover, by virtue of the close ties between CP non-unitary evolution and continuous generalized measurement processes, we used some results of the Quantum Measurement Theory [12]. The suitable generalization of the Pancharatnam connection allows us to identify the dynamical and geometrical contents of the total phase between two states under CP non-unitary evolution. The results obtained reduce to the corresponding expressions for total, geometrical and dynamical phases for unitarily evolving mixed states.

\footnotetext{
* This work was partially supported by Fundação para o Amparo à Pesquisa do Estado de São Paulo, FAPESP, and Conselho Nacional de Desenvolvimento e Pesquisa, CNPq.
} 
Completely positive mapping: A mapping $\Phi$ between density operators $\rho$ and $\rho^{\prime}$ is said to be completely positive (CP) [9, 13, 14, 15, 16] iff it has the form $\rho^{\prime}=\Phi(\rho)=\sum_{n} W_{n} \rho W_{n}^{\dagger}$, where $\left\{W_{n}\right\}$ are operators acting on the Hilbert space of the system of interest. If $\Phi$ preserves the trace, the set $\left\{W_{n}\right\}$ constitutes a partition of the unity:

$$
\sum_{n} W_{n}^{\dagger} W_{n}=1
$$

A CP continuous time evolution $\Phi_{t}$ is governed by the dynamical generator [14, 15]

$$
\dot{\rho}=\frac{1}{i \hbar}[H, \rho]+\frac{1}{2} \sum_{n}\left(2 V_{n} \rho V_{n}^{\dagger}-\rho V_{n}^{\dagger} V_{n}-V_{n}^{\dagger} V_{n} \rho\right),
$$

where $H$ is a hamiltonian operator and $\left\{V_{n}\right\}$ is a set of operators on the Hilbert space of the system of interest. Eq. (2) is called the Lindblad equation.

A CP non-unitary continuous time evolution describes a non-selective or non-referring continuous measurement process. In other words, at each infinitesimal time interval $d t$, the system suffers a generalized measurement and its state, after $d t$, is given by

$$
\rho(t+d t)=\sum_{n} W_{n}(d t) \rho(t) W_{n}^{\dagger}(d t),
$$

where the measurement operators $\left\{W_{n}(d t)\right\}$ are

$$
\begin{aligned}
& W_{0}(d t)=1-\left(\frac{i}{\hbar} H+\frac{1}{2} \sum_{n>0} V_{n}^{\dagger} V_{n}\right) d t \\
& W_{n}(d t)=V_{n} \sqrt{d t}, n=1,2, \ldots
\end{aligned}
$$

In a non-selective or non-referring measurement, the corresponding outcome is ignored. Each measurement operator is associeted to one of the possible measurement outcomes. The null result, in particular, is associated to the operator $W_{0}(d t)$. A null outcome occurs when no "pointer" deflection or no "click" is recorded by the meter. In other words, a null outcome occurs when the meter state is not changed during the measurement process.

Phases and measurements in interferometry: Consider a conventional Mach-Zhender interferometer, as shown in Fig. 1. A beam of particles follows through the paths labelled as " 0 " and " 1 ". These paths define a two dimensional Hilbert space $\tilde{\mathcal{H}}=\{|\tilde{0}\rangle,|\tilde{1}\rangle\}$. The state vectors $|\tilde{0}\rangle$ and $|\tilde{1}\rangle$ represent wave packets moving along the arms of the interferometer. The action of the mirrors, beam-splitters and the phase $U(1)$ shift due the difference of paths are represented by the unitary operators

$$
\tilde{U}_{M}=\left(\begin{array}{cc}
0 & i \\
i & 0
\end{array}\right), \tilde{U}_{B}=\frac{1}{\sqrt{2}}\left(\begin{array}{cc}
1 & i \\
i & 1
\end{array}\right), \tilde{U}(1)=\left(\begin{array}{cc}
e^{i \chi} & 0 \\
0 & 1
\end{array}\right) .
$$

As shown in the Fig. 1, the beam enters in the "0" arm. The "incoming" state is $\tilde{\rho}_{\text {in }}=|\tilde{0}\rangle\langle\tilde{0}|$. The beam reaches the detector $D$ in the "outcoming" state $\tilde{\rho}_{\text {out }}=\tilde{U}_{B} \tilde{U}_{M} \tilde{U}(1) \tilde{U}_{B} \tilde{\rho}_{i n} \tilde{U}_{B}^{\dagger} \tilde{U}^{\dagger}(1) \tilde{U}_{M}^{\dagger} \tilde{U}_{B}^{\dagger}$. The intensity measured by the detector is proportional to the matrix element $\left\langle\tilde{0}\left|\tilde{\rho}_{\text {out }}\right| \tilde{0}\right\rangle$, i.e., $I \propto 1+\cos \chi$.

Following the formalism of Sjöqvist et al. [11], let us assume that the particles have internal degrees of freedom (e.g., spin or polarization). The corresponding $N$-dimensional state space is denoted by $\mathcal{H}_{i}$. The initial state of the internal degrees of freedom is represented by the operator $\rho_{i, 0}$. Moreover, let us assume that the mirrors and beam-splitters do not influence the internal state. In order to obtain the phase relation between states under $\mathrm{CP}$ non-unitary evolution, a meter $P$ is introduced into the "1" arm of the interferometer, as shown in Fig. 1. The meter performs measurements on the internal degrees of freedom of the particles that cross the interferometer. By virtue of the inclusion of the meter, we are compelled to consider a third system associated with the meter - the probe, whose state space is denoted by $\mathcal{H}_{p}$.

The evolution of the composed system particles + internal degrees of freedom + probe after the first beam-splitter is given by the operator

$$
\mathbf{U}=\left(\begin{array}{ll}
0 & 0 \\
0 & 1
\end{array}\right) \otimes U_{i p}+\left(\begin{array}{cc}
e^{i \chi} & 0 \\
0 & 0
\end{array}\right) \otimes \mathbf{1}_{i} \otimes \mathbf{1}_{p}
$$


where $U_{i p}$ is an unitary operator acting in the product space $\mathcal{H}_{i} \otimes \mathcal{H}_{p}$. This operator describes the pre-measurement stage. The operators assigned to the other devices of the interferometer are $\mathbf{U}_{B}=\tilde{U}_{B} \otimes \mathbf{1}_{i} \otimes \mathbf{1}_{p}, \mathbf{U}_{M}=\tilde{U}_{M} \otimes \mathbf{1}_{i} \otimes \mathbf{1}_{p}$. Here, $\mathbf{1}_{i}$ and $\mathbf{1}_{p}$ are the identity operators on $\mathcal{H}_{i}$ and $\mathcal{H}_{p}$, respectively.

Without loss of generality, we assume that the probe is prepared in the pure state $\left|\zeta_{0}\right\rangle$. The state of the system particles + internal degrees of freedom + probe when the beam enters in the interferometer is thus $|\tilde{0}\rangle\left\langle\tilde{0}\left|\otimes \rho_{i, 0} \otimes\right| \zeta_{0}\right\rangle\left\langle\zeta_{0}\right|$. After the pre-measurement stage, the state of the global system is projected onto a vector belonging to a suitably chosen "pointer" basis of $\mathcal{H}_{p}$, namely $\left\{\left|\zeta_{n}\right\rangle\right\}_{n=0,1, \ldots}$. The intensity measured by the detector depends on the result of the measurement. In the case of a non-null result, we have

$$
I_{n} \propto \operatorname{tr}\left(W_{n}^{\dagger} W_{n} \rho_{i, 0}\right), W_{n} \equiv\left\langle\zeta_{n}\left|U_{i p}\right| \zeta_{0}\right\rangle, n=1,2, \ldots
$$

i.e., the interference pattern vanishes. In fact, a non-null result turns the paths distinguishable and destroys the interference pattern. If a null result is obtained, the intensity measured by the detector is

$$
I_{0} \propto 1+\operatorname{tr}\left(W_{0}^{\dagger} W_{0} \rho_{i, 0}\right)+2 \nu_{0} \cos \left(\chi-\phi_{0}\right), W_{0} \equiv\left\langle\zeta_{0}\left|U_{i p}\right| \zeta_{0}\right\rangle
$$

where we have defined the visibility $\nu_{0}=\left|\operatorname{tr}\left(W_{0} \rho_{i, 0}\right)\right|$ and the phase shift $\phi_{0}=\arg \operatorname{tr}\left(W_{0} \rho_{i, 0}\right)$. Therefore, only the null outcome produces non-vanishing visibility and contributes to form an interference pattern.

Finally, in the case of a non-selective measurement the intensity measured is given by the sum of the intensities $I_{n}$ :

$$
I=\sum_{n} I_{n} \propto 1+\nu_{0} \cos \left(\chi-\phi_{0}\right) .
$$

The limit of continuous measurements: In order to introduce time into the non-unitary dynamics, we assume that the meter $P$ performs repeatedly generalized measurements on the internal degrees of freedom. Each measurement takes a time interval $\Delta t$. Hence, in a unique measurement, the internal degrees of freedom unitarily interact with the probe and this interaction is described by the operator $U_{i p}(\Delta t)$. After this stage, the state of the global system particles + internal degrees of freedom + probe is projected onto a state of the basis $\left\{\left|\zeta_{n}\right\rangle\right\}_{n=0,1, \ldots}$ chosen in $\mathcal{H}_{p}$. Finally, the state of the probe is restored to the "quiescent" state (reset of the meter) and the process is repeated. The limit of continuous measurement is obtained taking $\Delta t \rightarrow 0$ and $N \rightarrow \infty, N \Delta t=t$.

Assuming that the initial state of the internal degrees of freedom is $\rho_{i}(0)$ and the quiescent state of the probe is $\left|\zeta_{0}\right\rangle$, the beam reaches the detector $D$ in the state

$$
\begin{aligned}
\rho_{\text {out }}(t)= & \frac{1}{4}\left\{\left(\begin{array}{cc}
1 & i \\
-i & 1
\end{array}\right) \otimes \rho_{i}(0)+\left(\begin{array}{cc}
e^{i \chi} & -i e^{i \chi} \\
-i e^{i \chi} & -e^{i \chi}
\end{array}\right) \otimes\left[\rho_{i}(0) S^{\dagger}(t)\right]\right. \\
& \left.+\left(\begin{array}{cc}
e^{-i \chi} & i e^{-i \chi} \\
i e^{-i \chi} & -e^{-i \chi}
\end{array}\right) \otimes\left[S(t) \rho_{i}(0)\right]+\left(\begin{array}{cc}
1 & -i \\
i & 1
\end{array}\right) \otimes \rho_{i}(t)\right\},
\end{aligned}
$$

where we have defined

$$
S(t)=\exp \left[-\left(\frac{i}{\hbar} H+\frac{1}{2} \sum_{n>0} V_{n}^{\dagger} V_{n}\right) t\right]
$$

It is worthwhile to remark that the evolution of the density operator $\rho_{i}(t)$ obeys the Lindblad equation (2).

The intensity measured by the detector $D$ is

$$
I \propto 1+\nu \cos [\chi-\phi(0, t)]
$$

where we have defined the visibility of the interference pattern

$$
\nu=\left|\operatorname{tr}\left[S(t) \rho_{i}(0)\right]\right|
$$

and the total phase difference between the states $\rho_{i}(0)$ and $\rho_{i}(t)$

$$
\phi(0, t)=\arg \operatorname{tr}\left[S(t) \rho_{i}(0)\right] .
$$


Parallel transport: Let us suppose that a quantum system initially prepared in the state $\rho_{0}=\rho(0)$ evolves nonunitarily during a time interval $t$. This evolution is $\mathrm{CP}$ and the dynamical generator has the form (2). The total phase between $\rho(t)$ and $\rho_{0}$ is

$$
\phi(0, t)=\arg \operatorname{tr}\left[S(t) \rho_{0}\right]
$$

We can employ the parallel transport condition to obtain the purely geometrical contribution to the total phase $\phi$ [17. In fact, the phase between the states $\rho(t+d t)$ and $\rho(t)$ is $\phi(d t)=\arg \operatorname{tr}\left[W_{0}(d t) \rho(t)\right]$, where $W_{0}(d t)$ is given by (3). Hence,

$$
\phi(d t)=\arg \left\{1-\frac{d t}{2} \sum_{n>0} \operatorname{tr}\left[V_{n}^{\dagger} V_{n} \rho(t)\right]+\frac{d t}{i \hbar} \operatorname{tr}[H \rho(t)]\right\} .
$$

$\rho(t+d t)$ and $\rho(t)$ are in phase if $\phi(d t)=0$. Note that

$$
1-\frac{d t}{2} \sum_{n>0} \operatorname{tr}\left[V_{n}^{\dagger} V_{n} \rho(t)\right] \geq 0
$$

since

$$
0 \leq \sum_{n>0} V_{n}^{\dagger} V_{n} d t \leq \sum_{n} W_{n}^{\dagger}(d t) W_{n}(d t)=\mathbf{1}
$$

Therefore, in order to satisfy the parallel transport condition it is necessary that

$$
\operatorname{tr}[H \rho(t)]=0
$$

This result is the analogue of the condition (12) of 11] for the parallel transport of mixed states under unitary evolution. Hence, if a state suffers a parallel displacement along a CP non-unitary curve $\Gamma: \tau \in[0, t] \rightarrow \rho(\tau)$ between the extreme points $\rho(0)$ and $\rho(t)$ such that Eq. (11) is satisfied, the total phase $\phi(0, t)$ has a purely geometrical character, i.e., $\gamma_{g}(\Gamma)=\phi(0, t)$. However, the condition stated in above equation is not sufficient. In the context of CP maps, a sufficient criteria is given by Eq. (20) of Ref. [18]. We can define the dynamical phase as

$$
\gamma_{d}(0, t)=-\frac{1}{\hbar} \int_{0}^{t} d \tau \operatorname{tr}[\rho(\tau) H]
$$

Eq. (10) constitutes the generalization of the Pancharatnam connection for quantum states undergoing a CP non-unitary evolution. In fact, in the unitary limit, the Eqs. 10 12 reduce to the expressions obtained by Sjöqvist and co-workers.

Example: Let us consider a single mode of the electromagnetic field undergoing a continuous and destructive photocounting process. The evolution of the field state $\rho$ is governed by the master equation

$$
\dot{\rho}=-i \omega\left[a^{\dagger} a, \rho\right]+k\left(2 a \rho a^{\dagger}-a^{\dagger} a \rho-\rho a^{\dagger} a\right) .
$$

The constant $k$ represents the photo-counting rate per time unit and $\omega$ stands for the mode frequency. Comparing the master equation (13) with the general form of the quantum dynamical generator (2), we have

$$
V_{1}=\sqrt{2 k} a
$$

and

$$
H=\hbar \omega a^{\dagger} a
$$

The master equation (13) describes the time evolution of a damped harmonic oscillator at zero temperature. If the mode is prepared in the initial coherent state $\rho(0)=|\alpha\rangle\langle\alpha|$ it is easy to verify that, after a time interval $t$, the state of the mode will be $\rho(t)=\left|\alpha e^{-(i \omega+k) t}\right\rangle\left\langle\alpha e^{-(i \omega+k) t}\right|$. According to Eq. (9), the total phase between $\rho(0)$ and $\rho(t)$ is

$$
\phi(0, t)=-|\alpha|^{2} e^{-k t} \sin \omega t
$$


and the dynamical phase is

$$
\gamma_{d}(0, t)=-\frac{\omega}{2 k}|\alpha|^{2}\left(1-e^{-2 k t}\right) .
$$

The geometric phase is given by the expression

$$
\gamma_{g}(0, t)=-|\alpha|^{2}\left[e^{-k t} \sin \omega t-\frac{\omega}{2 k}\left(1-e^{-2 k t}\right)\right] .
$$

The master equation (13) remains invariant under the transformations

$$
\begin{aligned}
& H^{\prime} \rightarrow H-\frac{i \hbar}{2}\left(\beta^{*} V_{1}-\beta V_{1}^{\dagger}\right), \\
& V_{1}^{\prime} \rightarrow V_{1}+\beta,
\end{aligned}
$$

The new measurement operators $W_{0}^{\prime}(d t)$ and $W_{1}^{\prime}(d t)$ describe a continuous homodyne photo-counting process [19]. The c-number $\beta$ is assigned to the amplitude of the local oscillator. Surprisingly, $\gamma_{g}^{\prime}(0, t)=\gamma_{g}(0, t)$. This result suggests that the geometric content of the phase between two states of a quantum system is invariant under unitary transformations of the measurement operators, since the non-selective evolution is not affected [20].

It is worthwhile to note that the phase space of the set of coherent states of the harmonic oscillator coincides with the complex plane. An initial coherent state remains coherent during the time evolution generated by Eq. (13), although its amplitude is damped. The geometric phase calculated in the two cases (conventional and homodyne measurements) is proportional to the area in the complex plane limited by the trajectory described by the time-dependent amplitude $\alpha(\tau)=\alpha e^{-(i \omega+k) \tau}$ and the shortest geodesic linking the extreme points, as shown in the Fig. 2.

Conclusion: We have obtained an extension of the notion of phases in interferometry for mixed states under CP non-unitary evolution. We have determined the dynamical and geometrical parts of the total phase between states undergoing a CP non-unitary evolution. Our results reduce to the corresponding expressions of Sjöqvist et al. [11] in the unitary limit.

[1] M. V. Berry, Proc. Roy. Soc. London A 392, 45 (1984).

[2] B. Simon, Phys. Rev. Lett. 51, 2167 (1983).

[3] F. Wilczek and A. Zee, Phys. Rev. Lett. 52, 2111 (1984).

[4] Y. Aharonov and J. Anandan, Phys. Rev. Lett. 58, 1593 (1987).

[5] J. Samuel and R. Bhandari, Phys. Rev. Lett. 60, 2339 (1988).

[6] S. Pancharatnam, Proc. Indian Acad. Sci. A 44, 247 (1956), reprinted in Geometric Phases in Physics, eds. A. Shapere and F. Wilczek, Advanced Series in Mathematical Physics 5 (World Scientific, Singapore, 1989).

[7] N. Mukunda and R. Simon, Ann. Phys. (N.Y.) 228, 205 (1993).

[8] For review of quantum theory of measurement, see P. Busch et al., The Quantum Theory of Measurement (Springer, Berlin, 1996). See also a recent treatment in A. Peres, Phys. Rev. A 61, 022116 (2000).

[9] For a rigorous treatment of the quantum open systems, see E. B. Davies, Quantum Open Systems (Academic Press, New York, 1976).

[10] A. Uhlmann, Rep. Math. Phys. 24, 229 (1986); id., ibd. 36, 461 (1995); id., Lett. Math. Phys. 21, 229 (1991).

[11] E. Sjöqvist et al., Phys. Rev. Lett. 85, 2845 (2000); LANL e-print quant-ph/00050702v2.

[12] In a context related with continuous measurements and the quantum Zeno effect, geometric phases for pure states undergoing non-unitary evolution are studied in A. K. Pati and S. V. Lawande, Phys. Lett. A 223, 233 (1996).

[13] A. Săndulescu and H. Scutaru, Ann. Phys. 173, 277 (1987).

[14] V. Gorini et al., J. Math. Phys. 17, 821 (1976).

[15] G. Lindblad, Comm. Math. Phys. 48, 119 (1976).

[16] W. F. Stinespring, Proc. Am. Math. Soc. 6, 211 (1955).

[17] For an alternative way to obtain a compact formula for geometric phase, see A. K. Pati, J. Phys. A: Math. and Gen. 28, 2087 (1995); id., Phys. Rev. A 52, 2576 (1995).

[18] M. Ericsson et al., LANL e-print quant-ph/0205160 2.

[19] H. Carmichael, An Open Systems Approach to Quantum Optics (Sringer-Verlag, Berlin, 1993).

[20] M. A. Nielsen and I. L. Chuang, Quantum Computation and Quantum Information (Cambridge University Press, Cambridge, 2000); J. Preskill, lecture notes, www.theory.caltech.edu/ preskill/ph229; K. Jacobs, PhD Thesis, LANL e-print quant-ph/9810015 


\section{Figure captions}

Fig. 1. A conventional Mach-Zhender interferometer. $M_{1}$ and $M_{2}$ are perfect mirrors, $B_{1}$ and $B_{2}$ are balanced beam-splitters. The detector $D$ measures the intensity of the beam of particles in " 0 " arm. $\chi$ is the $U(1)$ phase shift due the path difference. In order to introduce non-unitary evolution, a meter $P$ is connected into " 1 " arm.

Fig. 2. The geometric phase between the coherent states $\rho(0)=|\alpha\rangle\langle\alpha|$ and $\rho(t)=\left|\alpha e^{-(i \omega+k) t}\right\rangle\left\langle\alpha e^{-(i \omega+k) t}\right|$ of the harmonic oscillator evolving according to the master equation (13) is proportional to the area of the region in complex plane limited by the trajectory described by the time-dependent amplitude $\alpha(\tau)=\alpha e^{-(i \omega+k) \tau}, \tau \in[0, t]$, and the shortest geodesic linking the extreme points. 